If the patient died, I should be still more on my guard. And I would urge the same humane precautions on every practitioner. I felt it to be my duty to discontinue midwifery for a fortnight, which my medical brethren in the town kindly took charge of.

\section{OBSERVATIONS ON SPERMATORRHQEA;} OR

THE INVOLUNTARY DISCHARGE OF THE SEMINAL PLUID.

By W. H. Ranxing, M.D., Cantab, Physieian to the suffolk General Bospital.

(For the Provinoial Medical Journal.) (Continued from page 29.)

Diagnosis. - There is no difficulty in general in recognising the existence of spermatorrhœa, the presence of continual nocturnal emissions leaving no room for misapprehension. But in the more aggravated forms of the disease there arises a complication of symptoms, which, taken in connection with the disappearance of the nocturnal losses, gives rise to frequent mistakes. These cases, in which the seminal fluid drains away in the evacuations of the bladder and rectum, are usually confounded with chronic affections of the brain and alimentary canal, to which they bear so olose a resemblance, as to require considerable tact for their discrimination, especially when, as is often the case, the patient obstinately refuses to make his medical attendant the confidante of his habits of life. Had we any positive signs by which we could recognise the presence of the seminal fluid in the urine, the diagnosis would be perfectly simple; but, unfortunately, such is not the case. There is so commonly an admixture of the morbid secretions from the bladder and prostate, together with amorphous urinary sediments, that neither the microscope or any other agent is sufficient to determine its existence. According to Lallemand, the presence of semen is to be suspected whenever the urine is voided thick and fœtid, and exhibits o cloud resembling thin gruel suspended in it. The seminal fluid, it is to be remembered, generally passes away toward the completion of the act of micturition. Where the fluid is discharged during defecation, there is no difficulty in its detection, as by the assistance of the patient it may be submitted to ocular demonstration.

But, setting aside this desirable but at present unattainable proof of the existence of semen in the urine, let us inquire whether there are any general grounds for distinction between the cerebral symptoms produced by spermatorrhœa and those which depend upon idiopathio disease of the brain. The following considerations will enable us to arrive at something like a satisfactory conclusion upon the subject. In the case of spermatorrhcea, the cerebral phenomena are not the first in the chain of symptoms, but are preceded for a considerable time by notable derangement of other functions of the body; the digestion will be found to have been previously impaired, and the sexual vigor usually fails in a degree disproportionate to the general debility. In idiopathic cerebral disease, on the contrary, the symptoms of disordered circulation in the brain-as giddiness, tinnitus aurium, dimness of sight, \&c.-take precedence of the disturbance in other organs; the appetite and digestion are not necessarily impaired, neither is the sexual vigor observed to decline until an advanced stage of the disease. There is not, moreover, the extreme emaciation and feebleness of spermatorrhœa, but the general powers of the body fail only in proportion to the increase of the cerebral disorder. In doubtful cases I consider that the condition of the urinary and genital functions is our best guide in diagnosis. Wherever we have frequent micturition of turbid urine (calculous cases excepted), combined with the gradual extinction of the procreative powers, whatever be the accompanying symptoms, we shall do right to direct our inquiries to the condition of the seminal apparatus.

Having decided upon the existence of seminal losses, the next question is, to determine whether it depends upon chronic inflammation or upon simple atony of the parts. This question can only be decided mainly by the history and general appearance of the patient, as the more prominent symptoms are identical in the two cases. It is, nevertheless, of considerable importance regards treatment, for a misapprehengion of the pathology will lead to oonsiderable waste both of time and credit. 'l'he following are the reasons which should lead to the supposition of simple debility of the seminal vessels.

These are signs of general congenital feebleness, as small osseous and muscular development, with late puberty, thin whitish hair, slight appearance or total deficiency of beard, and high treble tone of voice. In addition to these signs of sexual feebleness may be mentioned a general roundness of contour, with wide hips and full thighs. In the genital organs themselves may be observed smallness and flaccidity of the penis, which appears to be drawn close up to the pubes, an elongated prepuce, and small pendulous testicles. I have, while engaged upon this paper, seen a severe case of seminal emissions, depending upon debility, in which one testicle only has descended in to the scrotum. Congenital hernia and varicocele are also to be considered as evidences of a debilitated sexual apparatus, as is likewise the presence of incontinence of urine in childhood. If, on the contrary, the patient is of a well-developed frame, with genital organs of normal dimensions, if he has at one time enjoyed arerage sexual power, we shall be authorised in regarding the complaint a one of chronic inflammation, whatever be the degree of accompanying emaciation and feebleness.

Treatment.-In noticing the treatment of seminal emissions we dismiss entirely those which occur but seldom, and in robust continent individuals, since, as has been before stated, such discharges are not to be considered morbid. The following remarks are to be viewed as referable to diumal and nocturnal pollutions of frequent recurrence.

Our first object must be to inform ourselves of the exciting cause, and at once to remove it. If the emission coexist with ascarides in the rectum, our treatment must be directed to their expulsion, upon which, in some cases, the patient will be restored to health without the intervention of other means. The ordinary remedies for these worms are sufficientsuch as calomel, purges, salt and water injections, and mercurial inunction of the anus. When the complaint depends upon disease of the rectum inducing constipation, as stricture, fissure, or homor- 
rhoids, the appropriate surgical treatment is to be employed. Sebaceous secretion behind the prepuce is, as I have before said, an occasional exciting cause. This is to be met by cleanliness and the use of astringent washes. If, as is sometimes the case, this morbid secretion is retained by an elongated and narrow prepuce, circumcision is the obvious remedy. In some cases the mere removal of these exciting causes will be sufficient to effect a cure, but in the majority a morbid condition of the spermatic ducts has been established, which must be combatted by means hereafter to be described.

The treatment of that form of the disease which coexists with general debility is to be attempted by such means as are likely to improve the tone of the system. In these cases the treatment which is too often followed indiscriminately-viz., cold bathing and cold douches upon the parts-will in general be of great utility. The douche should be used at least three times a-day, and continued each time until an unpleasant sensation of cold is produced. In conjunction with these means, tonics, especially the mineral forms, should be exhibited. The tinctura ferri sesquichloridi is, perhaps, the best. It will occasionally be found that the nocturnal emissions are at first increased in frequency under this treatment, but we are not to be dispirited at such an effect; it is only temporary in its duration, and the emissions produced under the influence of the medicine are not nearly so enfeebling as those which occur previously to its employment. It is a question whether sexual intercourse should be recommended in these cases. Putting religious considerations aside, the opinion of Lallemand appears to be rational, that the occasional employment of the organs would conduce to their vigor, upon the principle that a weakly frame generally is strengthened by the moderate employment of the exercise specially adapted to each of its parts.

Such is the simple mode of treating that form of spermatorrhœa which is connected with general debility, and depends upon an atonic state of the spermatic vessels; we have now to consider the treatment of the most important form of the diseasethat which has for its exciting cause undue exertion of the sexual organs, and which depends essentially upon chronic inflammation of the parts concerned.

In the first place, the excess which produced the disease must be abolished; and here is the principal difficulty: so firm a hold has the pernicious vice which is generally observed to be indulged in, that inducements or threats are often alike inefficacious in preventing it. Sexual intercourse must be forbidden; unlike the other form of the disease, in this it is positively hurtful. The great error in the treatment of these cases lies in the endeavour to support the debilitated frame by nourishing food and stimulating drinks. Wine and beer must not be allowed, neither must the food be highly seasoned. The diet should, on the contrary, be of the simplest kind-as milk and bread, and well-cooked and digestible vegetables. The drink water. The application of cold is of doubtful efficacy in these cases; some, indeed, are aggravated in a notable degree after cold bathing and the douche. The method upon which the greatest reliance is to be placed for the absolute cure of the disease is the application of nitrate of silver to the affected portion of the urethra. It is not my intention to discuss the manner in which this remedy acts, suffice it to say that its power in modifying the sensibility and restoring the functions of diseased mucous membranes is now a matter of every day notoriety.

The application of lunar caustic to the urethra in cases of spermatorrhœa was first the suggestion of Lallemand; for although Sir E. Home had previously cauterised the canal, it was with the object of overcoming a stricture. Lallemand prefers in all cases the application of the solid nitrate; but it may likewise be used with benefit in the form of solution. In the hands of the French surgeon the success of this mode of treating involuntary emissions has been most remarkable; nearly one hundred cases are reported in which it was adopted, and in all, with very few exceptions, its effects have been rapid, and where the patient has been commonly prudent, subsequently to his treatment, permanent. The experience of British surgeons, though not so extensive, is, as far as it goes, equally satisfactory. In the excellent critique upon Lallemand's works in the "Brit. and For. Med. Review" (No.30), are collected the written testimony of several English practitioners. One gentleman writes thus: "I can recollect eleven cases in which I have found Lallemand's treatment successful, and one in which it did not completely succeed. In seven of the eleven cases a single application of the caustic was enough, in four it was necessary to apply it a second time. The effects are immediate. A person in whom the discharge has continued for months will have none for some days after the use of the caustic." Another writes to the following effect: "I have carefully noted twenty-seven cases treated by the nitrate of silver; of these, thirteen were completely cured ; eight so much benefitted that the emissions only recurred occasionally, and produced but little effect on the sys. tem; the remaining five were benefitted, but not to the same extent. A third surgeon states that, "with regard to Lallemand's method of cauterising the urethra, he has tried it in a dozen cases, and in the majority of them with decidedly good effects." Mr. Phillips, in a paper above alluded to, thus speaks of the caustic bougie, "In nineteen cases I used the caustic. Of these cases, ten were completely relieved by a single application; in three the amelioration was decided, though the complaint was not cured; in six there was no relief; in the nine cases in which the first application was insufficient the remedy was again used, in three cases with complete relief." So that " in thirteen cases out of nineteen it succeeded, in six it failed, but in no case was there any aggravation." It appears, then, from these remarks, that of fifty-eight cases, exclusive of those of Lallemand's, in which caustic was applied, it failed only in six, was beneficial in fourteen, and completely successful in thirty-seven, or two-thirds-a result sufficient to establish its character as a remedy of the utmost value.

In the majority of the cases one application of the caustic is sufficient, and more than a second is seldom necessary. No evil effects are produced by it, beyond those of transientirritation - such as scalding on making water, with occasionally a slight admixture of blood. My own experience of the remedy is not extensive; I have caused it to be tried only in three cases; in all 
with perfect success. One case was of the utmost severity, and as it affords an excellent example of the affection, and pourtrays the obstinacy with which it resists ordinary means of cure, I think it may not be uninteresting to relate. $\mathrm{Mr}$. - $\longrightarrow$, aged nineteen, of moderately robust original constitution, consulted me about a year since in the following condition. He had emaciated to a considerable extent, and had become utterly incapable of exertion; the slightest exercise induced fatigue and profuse perspiration. His face was pallid, expressive of timidity, and covered with acne indurata and punctata. His mind had become enfeebled; he was unable to read with attention, and wept upon the most trivial occasions. He complained of anomalous pains in various parts of the body, and suffered severely from gastrodynia. He had frequent attacks of hysteric delirium, accompanied with excessive palpitation. The bowels resisted obstinately large and repeated doses of croton oil. Nocturnal emissions had existed for many months, and at this time occurred several times during many successive nights, leaving him in a state of the utmost moral and physical prostration. The mere act of conversing with a female induced a discharge, but he had lost all power of erection.

The genital organs were well developed; but the testicles were tender upon pressure, and pendulous. He confessed to the long and frequently indulged habit of masturbation, but, as is commonly the case in such persons, had committed no excesses with women. He had been treated by more than one practitioner in the usual way-that is, by tonics and good living-without avail. As he complained of considerable pain along the perineum, I commenced the treatment by leeches, and with good effect as to the pain, but without diminishing the frequency of the emissions. I next ordered an injection of four grains to the ounce of nitrate of silver three times a-day, under which the emissions were suspended for several days together, and he gained a corresponding amount of strength. I lost sight of him from this time till January last, when I was summoned to him suddenly, and found that he was as bad as ever, and in a state of complete hysteria; I determined, therefore, to cauterise immediately. A surgical friend having first passed a common bougie, we found the membranous portion of the urethra highly sensitive, and cauterised it by means of Lallemand's instrument. Very little pain was complained of. In a few days an alteration had taken place in the patient which surprised me; he had had but one emission since the operation, could retain his water, and had experienced erection of the penis, which had not occurred for months. A daily improvement took place; his mind became clear, and capable of attention; his procreative powers returned with vigor, and he is now in perfect health.

The method of performing the operation is as follows :-A common bougie or catheter is to be passed in the first instance, and the length is to be noted at which the urine begins to flow; an inch short of this will give the situation of the membranous portion of the urethra. The armed instrument is then to be passed to the same length, and the caustic being denuded, it is to be made to revolve, by twirling the instrument for the space of an inch and a half, when it is to be drawn within the bougie and extracted.
Nothing can be more simple. If the ardor urinæ be excessive, and there be much pain after the operation, a hip bath may be used, but in general no ill consequences are observed.

As auxiliaries to cauterisation in the treatment of involuntary emissions, the occasional application of leeches will be found useful. The patient should also be recommended to lie upon a hard mattrass, and never, if possible, to sleep upon the back, as pollutions are, beyond a doubt, more likely to occur in that position than in any other. The use of a cold enema, with twenty drops of laudanum upon going to bed, will greatly assist in preventing them.

Much care is requisite in managing the convalescence of these patients. Their newly acquired sexual vigor is apt to lead them to a repetition of excess, by which a relapse is most readily induced. As soon as the local irritation has been subdued, a course of tonics may be recommended.

Bury St. Edmunds, Sept. 21.

\section{PROVINCIAL MEDICAL JOURNAL}

\section{SATURDAY, NOVEMBER 4, 1843.}

We have more than once directed the attention of our readers to the deplorable results of that random administration of powerful remedies which occurs daily and hourly in the shops of chemists who prescribe for their ignorant customers, and we have pointed out the fraudulent uses which many chemists are making of their newly-acquired diploma from the Pharmaceutical Society, by causing it to be considered by the ignorant as a qualification for medical practice. We think it right to inform our readers occasionally of the movements of this society, because, whatever may be the conscientiousness of its founders, or their desire to prevent their associates from making an improper use of their so-called testimonial of qualification, it must be recollected that they can have but a very slight control over a body of tradespeople, most of whom cannot afford to be particularly scrupulous.

It will be difficult to deny that, although the indiscriminate distribution of the diploma to all applicants may be an admirable means of filling the coffers of an infant institution, and of obtaining that influence which is always produced by numbers, be they who they may, yet that it is not an equally certain means of ensuring (for at least a generation) those high objects which the founders have at heart-that is to say, the respectability, education, and quasi-professional character of the members. Whatever education may be required from future candidates, whatever examination they may be submitted to, they fall back undistinguished into the general illiterate mass, who have obtained the same diploma by priority of application and by purchase.

We need hardly adduce much evidence to prove to 